Original Article

Dr. Reema Rajbhandari

MD, Assistant Professor

Dr. Parmatma Prajuli

MD Lecturer

Dr. K K Oli

PHD, Professor

Department of Neurology, Tribhuvan University

Teaching Hospital

Address for correspondence:

Dr.Reema Rajbhandari, MD

Assistant Professor. Department of Neurology.

Tribhuvan University Teaching Hospital.

Kathmandu Nepal

Email: reemarajbhandari@hotmail.com

Contact No :- +977-9841886489

Date submitted : 23/3/2019

Date accepted : 7/5/2019

\section{Ischemic Stroke in Young Adults: Study of the Subtypes, Risk factors and Predictors of Poor Outcomes}

The aim of this study is to show the subtype, risk factors and predictors of poor outcomes in young ischemic stroke patients.

Materials and Methods: It is a prospective study where only young onset ischemic stroke patients are analyzed.

$8 \%$ of total ischemic stroke sufferers were young adults; more prevalent in female (57.1\%), with risk factors of smoking (47.6\%) subtype cardio embolic (42.9\%) was common. High NIHSS score was related to new event and correlation to mortality. Three month follow up showed MRS $2.86 \pm 1.02 .57 .1 \%$ of cases had significant disability (defined as MRS $\geq 3$ ). There was a positive correlation of the cardio embolic subtype and the unclassified subtype to all three forms of poor outcomes, along with a positive correlation of the large artery atherothrombotic subtype to new events Recurrence of events (new stroke, sudden increase in symptoms and new TIAs) was observed in $14.3 \%$. Death was reported in $9.5 \%$ of cases.

Etiological diagnosis can be reached in majority of cases if an aggressive approach is maintained. This small scale study has provided an overview of the distribution of subtypes, risk factors and poor outcomes and their predictors. Cardiac diseases (esp. valvular disease) need to be taken more seriously to prevent cardio embolic strokes, while addressing other traditional risk factors to prevent the atherothrombotic subtypes.

Keywords: Ischemic stroke, Young Adults, subtypes, outcomes

hospital in Nepal, was designed to identify etiological subtypes of ischemic stroke, their relation to risk factors, and predictors of its outcome, which is fundamental in addressing the problem diligently, with effective preventive and therapeutic strategies.
Ischemic stroke is the third leading cause of death in Nepal. This prospective study, based in a tertiary teaching oung adults 15 to 45 years, who are of economically productive age group suffering from ischemic stroke is a major health hazard. 


\section{Rajbhandari et al}

\section{Material and Methods}

All cases of ischemic stroke with age 15 to 45 presenting to Tribhuvan University Teaching Hospital (TUTH), a tertiary care center in Nepal, within 72 hours of onset were enrolled in a prospective registry starting from May1 2013 December 2013. The diagnosis of ischemic stroke was based on clinical features and imaging in all patients. Each patient underwent a clinical neurological examination, documentation of NIHSS scores and a stroke work up: imaging Computed Tomography (CT), Magnetic Resonance Imaging (MRI), Doppler ultrasound of major neck arteries, Electrocardiography monitoring (ECG), standard laboratory blood tests and trans esophageal or transthoracic echocardiogram in cases with no obvious stroke etiology after routine assessment, coagulation and vacuities profile were also obtained. Documentation of patient characteristics, conventional risk factors (family history of stroke, smoking, obesity, diabetes mellitus, hyperlipidemia, hypertension, coronary artery or peripheral vascular disease) together with medical history, laboratory and technical investigations and severity (NIHSS scores) were carried out according to standard protocol. 16 Trial Of ORG 10172 in Acute Stroke Treatment (TOAST) classification system was used to classify strokes according to etiology. ${ }^{1,9}$ All cases were followed up for up to a minimum of three months after discharge to assess and record outcomes. Poor outcomes were defined in terms of significant disability (a modified Rankin score of more than 2), new cerebrovascular events i.e. new stroke or TIA and mortality. Permissions from the Institutional Review Board and the Ethics Committee were obtained prior.

All statistical analyses were carried out with SPSS version 20.0. Bivariate correlation studies and Factor analysis were obtained to assess the degree of association of risk factors, symptoms at presentation, early disability, in hospital morbidity, stroke severity to subtypes of stroke and poor stroke outcomes. Positive relations were reexamined with ANOVA. All associations with a $p$ value of $<0.05$ were considered significant.

\begin{tabular}{|l|l|}
\hline Parameter & Value \\
\hline Age mean \pm s.d. & $32.86 \pm 9.7$ years \\
\hline Range & $15-45$ years \\
\hline Females & 12 cases $(57.1 \%)$ \\
\hline From inside Kathmandu valley & 11 cases $(52.38 \%)$ \\
\hline Agriculture related & $13 \operatorname{cases}(61.9 \%)$ \\
\hline Manual labourer & 2 cases $(9.5 \%)$ \\
\hline
\end{tabular}

\begin{tabular}{|l|l|}
\hline Business related & 2 cases $(9.0 \%)$ \\
\hline Student & 4 cases $(19 \%)$ \\
\hline Presentation inside first 3 & 6 cases $(28.6 \%)$ \\
\hline hours of onset & 7 cases $(33.3 \%)$ \\
Presentation inside first 6 hours of & \\
\hline $\begin{array}{l}\text { Mean time of presentation to the } \\
\text { emergency }\end{array}$ & $33.76 \pm 30.7$ hours \\
\hline
\end{tabular}

Table 1. Demographic characteristics

\section{Results}

\section{Stroke Risk Factors}

Ischemic strokes in young adults were more prevalent among females; males accounted for only $57.1 \%$ of the total cases. The older age range i.e. 35 to 45 years accounted for only $42.9 \%$ of cases. A history of smoking was present in only $47.6 \%$ of cases. In all of the smokers, the quantity of smoking (as measured by the number of pack-years of smoking) was less than $20.128 .6 \%$ of the cases had a history of alcohol consumption. A history of hypertension was present in only $19 \%$ of the cases. However, a measured blood pressure of more than or equal to $140 / 90 \mathrm{~mm}$ of $\mathrm{Hg}$ at admission was observed in $23.8 \%$ of the cases. A history of dyslipidemia was present in $14.3 \%$ of the patients. On evaluation post-admission, elevated serum LDL levels were observed in $19 \%$ of the cases and low HDL in another $9.5 \%$ of cases. A history of diabetes mellitus was present in $9.5 \%$ of the cases. Hyperglycemia (defined by random blood sugar $>10$ $\mathrm{mmol} / \mathrm{l}$ ), was observed during admission in $19 \%$ of the cases. Elevated HbA1c (more than 6.5\%) denoting long term hyperglycemia or poorly controlled diabetes mellitus was observed in none of the cases. Most of the cases were fairly active, physically, in their pre-morbid life with 61.8 $\%$ of cases carrying out household and agriculture related activities, $9.5 \%$ of the cases were manual laborers and 19 $\%$ of the cases had school and household related physical activities. Only $9.5 \%$ of the cases had a sedentary lifestyle. Only $14.3 \%$ of the cases could be labeled as over-weight with respect to their waist circumferences.

A past history of TIA or previous cerebrovascular accident was present in $14.3 \%$ of the cases. There was a past history of known rheumatic valvular heart disease (RHD) in $42.9 \%$ of the cases. Abnormal ECG was observed in $33.3 \%$ of the cases, having AF as their rhythm.

There were examination findings suggestive of collagen vascular disease/vasculitis in $14.3 \%$ of the cases. The 


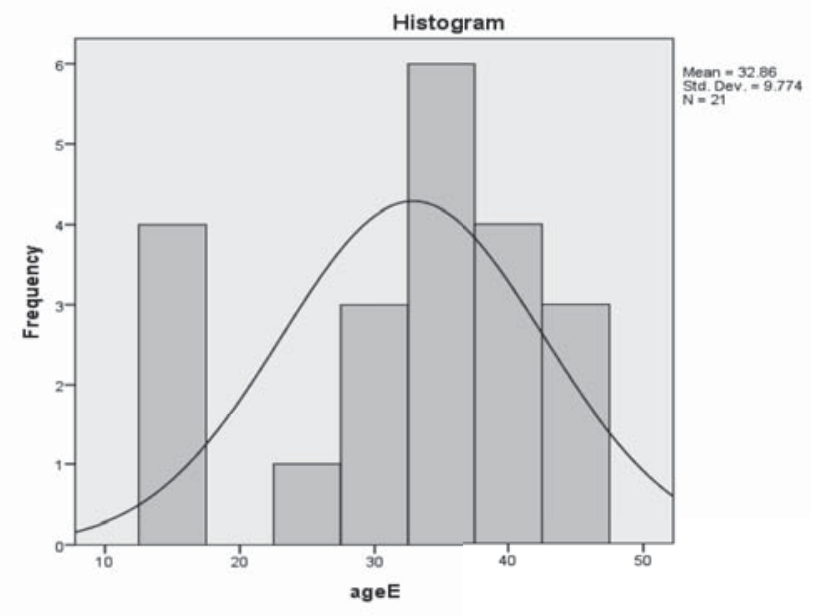

Table 2:Histogram of Age distribution.

findings were those of Takayasu's Arteritis and Systemic Sclerosis in $9.5 \%$ and $4.7 \%$ of cases respectively. $4.7 \%$ of the cases were positive for ANA.

\section{Stroke Subtypes}

The major subtype of the ischemic stroke (as per the TOAST classification system) was cardioembolic accounting for $42.9 \%$ of the cases. Small vessel atherothrombotic disease accounted for $9.5 \%$ of cases and another $23.8 \%$ of the strokes was due to vasculitis or pregnancy related which fall under the categoryunclassified. $19 \%$ of the cases were of the subtype large artery atherothrombotic. Stroke subtype could not be determined in $4.8 \%$ of the cases, thus, falling under the category-undetermined.

Regarding imaging characteristics, majority $(85.7 \%)$ of cases had a territorial pattern on imaging, denoting involvement of a single large vascular territory. Lacunar infarcts and multiple infarcts were observed less frequently ( $9.5 \%$ and $4.8 \%$ respectively).

\section{Outcomes}

The mean Modified Rankin Scale scores of the cases at 3 months of follow up were $2.86 \pm 1.02 .57 .1 \%$ of cases had significant disability (defined as MRS $\geq 3$ ). Recurrence of events (new stroke, sudden increase in symptoms and new TIAs) was observed in $14.3 \%$ of cases at 3 months of follow up. Death was reported in $9.5 \%$ of cases. All mortality was reported post-discharge and within 6 weeks of the initial event.

Relation of Stroke subtypes to Risk Factors

Analysis of the traditional risk factors with respect to the stroke subtypes showed majority of the traditional risk factors widely distributed throughout the subtypes.

There were some noteworthy associations

Cardioembolic strokes were seen to be significantly associated to male gender $(\mathrm{p}=0.003)$.

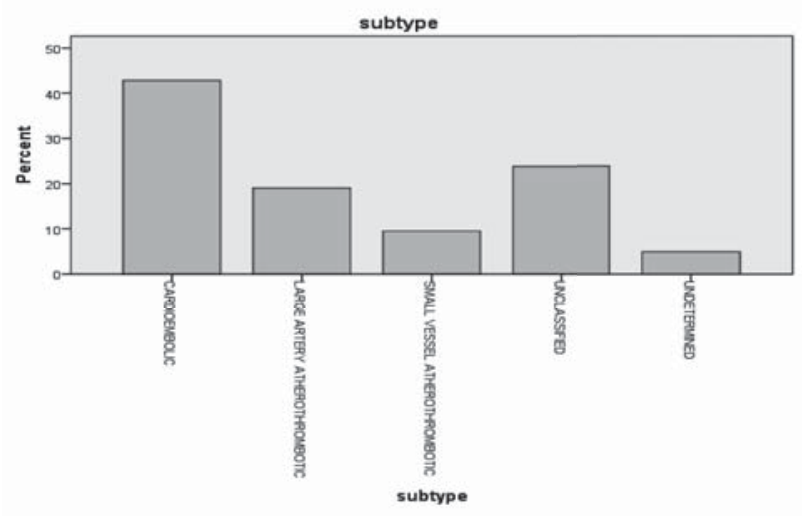

Table 3: Stroke subtype

Small vessel atherothrombotic strokes were observed to be related significantly to a past history of having transient ischemic attacks $(\mathrm{p}=0.025)$.

Large artery atherothrombotic disease was seen to be associated significantly to smoking $(\mathrm{p}=0.013)$.

The unclassified subtype of ischemic stroke was related to a history of having vasculitis $(p<0.001)$. There was an association of this form of stroke to the female gender $(\mathrm{p}=0.023)$.

\section{Stroke risk factors and severity}

Among all the traditional stroke risk factors, only male gender was found to have a statistically significant relation to increased stroke severity $(\mathrm{p}=0.012)$.

\section{Stroke subtype and severity}

Though cardioembolic strokes showed a trend towards being related to increased severity, no particular stroke subtype was significantly related to severity of the strokes.

\section{Stroke outcomes and traditional risk factors}

Poor stroke outcome in the form of significant disability (a modified rankin scale of above 2) was significantly related to having a history of hypertension $(\mathrm{p}=0.024)$, diabetes mellitus $(\mathrm{p}=0.003)$, dyslipidemia $(\mathrm{p}=$ $0.023)$ and a sedentary lifestyle $(\mathrm{p}=0.001)$. Recurrence of vascular events was faound to be associated most significantly to a sedentary lifestyle $(\mathrm{p}=0.008)$. A history of diabetes mellitus ( $p=0.022)$ and smoking $(p=0.02)$ were also significantly associated to new events.

Mortality was significantly related to a history of having a sedentary lifestyle $(\mathrm{p}=0.025)$. Though a history of hypertension and diabetes mellitus had a positive correlation to mortality, statistical significance was not achieved. 


\section{Rajbhandari et al}

\begin{tabular}{|l|l|}
\hline Morbidities post-admission & Prevalence \\
\hline Fever & $38.1 \%(8$ cases $)$ \\
\hline Pneumonia & $38.1 \%(8$ cases $)$ \\
\hline UTI & $14.3 \%(3$ cases $)$ \\
\hline DVT & $0 \%(0$ cases $)$ \\
\hline Heart failure & $23.8 \%(5$ cases $)$ \\
\hline
\end{tabular}

Table 4: Morbidities post admission.

\section{Stroke subtypes and poor outcomes}

Cardioembolic stroke and the Unclassified stroke subtypes had a positive correlation to all three forms of poor outcomes - disability (MRS $>2$ ), new events and mortality , and , large artery atherothrombotic subtype had a positive correlation to new events. All these relations however were not strong enough to achieve statistical association.

Abnormalities in serum sodium levels (hyponatremia or hypernatremia) were associated to post stroke disability $(\mathrm{p}=0.006)$ and mortality $(\mathrm{p}=0.022)$.

\section{Discussion:}

$8.07 \%$ of all ischemic strokes affected young adults ( 15 to 45 years of age) in our study, which is similar to the figures obtained from several other large stroke registries, which show a prevalence of ischemic stroke in young adults (of the above specified age group) to be between 4 to $12 \%$ of all ischemic strokes ..$^{1,2,3,4,5,17,18}$

Themostprevalentstrokesubtypewasthecardioembolic stroke subtype $(42.9 \%)$ followed by the unclassified subtype $(23.8 \%)$, the large artery atherothrombotic subtype (19\%), the small vessel atherothrombotic subtype ( $9 \%)$ and the undetermined subtype (4.8\%). This finding is different from other studies in which the major subtype is the undetermined subtype. 4 The larger prevalence of cardioembolic stroke in this study may reflect the greater prevalence of rheumatic heart disease in a developing nation like Nepal.

The findings that the undetermined subtype affects only $4.8 \%$ of all cases is acceptable, because, it indicates the degree of thoroughness attained in the investigations aimed to achieve an etiological diagnosis. The disparity in proportion of rheumatic heart disease (and consequently cardioembolic stroke in young adults) in our population and elsewhere might have contributed to the disparity in proportion of the undetermined stroke subtype.
The relatively more prevalent gender in our study is females $(57.1 \%)$. This finding in our study is in coherence with the inherent lower life-expectancy (and probably higher propensity of Nepalese women to have non communicable disease). Though females were affected more with strokes as a whole, male gender was associated with increased occurrence of cardioembolic strokes with a $p$ value of 0.003 . This is in contrast to the relative proportion of rheumatic heart disease in girls and boys. Other factors viz. the difference in severity of the underlying heart disease and association with rhythm disturbances, however, might play confounding role.

Smoking is prevalent in $47.6 \%$ of the cases in our study. In consistency to other studies, smoking is associated significantly with the large artery atherothrombotic subtype of stroke in our study $(\mathrm{p}=0.013) .{ }^{15,19}$

While a past history of having valvular heart disease was strongly predictive of the stroke being of the cardioembolic subtype $(\mathrm{p}<0.001)$ a past medical history significant for transient ischemic attacks (TIA) was associated significantly to the small vessel atherothrombotic subtype of ischemic stroke $(\mathrm{p}=0.02)$.

A medical history significant for hypertension, diabetes mellitus, dyslipidemia and a personal history of sedentary lifestyle were not seen to be associated significantly to any particular subtype of ischemic stroke including the atherothrombotic subtypes (large artery and small vessel) in particular, in contrast to their well-known and proven association to ischemic strokes in the older population.

Systemic vasculitis - Takayasu's arteritis was seen to be associated to the unclassified subtype of stroke along with systemic sclerosis $(\mathrm{p}<0.001)$. Their causal association to this particular subtype is also strengthened by the fact that raised ESR, a marker of active inflammation, was associated significantly to the unclassified subtype of ischemic stroke $(p=0.044)$. This subtype of ischemic 
stroke was related exclusively to females $(\mathrm{p}=0.023)$, the gender most likely affected by systemic vasculitis.

Regarding severity, majority of the ischemic strokes in young adults $(52.4 \%)$ were severe during hospital admission, as denoted by a higher NIHSS score $(>24)$. A similar proportion of cases $(52.4 \%)$ could be classified as having severe strokes depending upon a low Glasgow Coma Scale scores.

Cardioembolic stroke subtypes were the most severe at presentation as shown by their trend towards prevailing among those with a lower GCS and a higher NIHSS scores. It is consistent with the finding that cardioembolic strokes often lead to large territorial strokes in comparison to other subtypes. The relation was however not statistically significant, and thus, no particular subtype of ischemic stroke can reliably predict an increased severity of the illness.

The only stroke risk factor that was associated significantly to increased severity of stroke was male gender $(p=0.034)$, probably resulting from this gender's association to cardioembolic strokes. The severity of underlying heart disease may also play a confounding role.

No other traditional risk factors were seen to directly relate to severe illness, in contrary to what might be expected from data from the elderly populations.

Poor outcomes (in terms of morbidity/disability, new events and mortality) were of considerable magnitude in ischemic stroke in young adults. More than a half of all cases were disabled enough to require assistance for all forms of activities at three months of follow up. One in seven cases had a recurrence/appearance of a new event and just less than $10 \%$ of all cases succumbed to their strokes. Though our findings are similar to those from the sub-continent, they are in sharp contrast to the figures regarding outcomes from the developed nations — a reported mortality of less than $5 \% .{ }^{11}$ This difference highlights the difference in the standards of health care services. This finding also indicates that there is utmost need and plenty of room for further improvement.

In contrast to other studies, diabetes mellitus did not have a direct relation to mortality in our study. Sedentary lifestyle, among all, was seen to have a strikingly significant association to disability esp. to mortality. ${ }^{11}$

While choking/swallowing difficulty and slurring/ language difficulty were associated significantly to disability, mortality was associated to choking/ swallowing difficulty only may have a predictive value for poor prognosis. A low GCS during admission had a trend towards being associated to significant disability and mortality, a high NIHSS score was related to new events and had a correlation to mortality. This association of NIHSS scores is in consistency to findings in several other studies. ${ }^{11}$

Similar to the trend of association of ischemic stroke subtypes to the severity of illness, there was a positive correlation of thecardioembolicsubtypeand theunclassified subtype to all three forms of poor outcomes, along with a positive correlation of the large artery atherothrombotic subtype to new events. A significant statistical association could be obtained in none of these subtypes with respect to poor outcomes, however, precluding them to be proposed /used as predictors of poor prognosis.

A strikingly significant association was observed between abnormalities in the serum levels of sodium (both hyponatremia and hypernatremia) to all three forms of poor outcomes. With respect to the strong degree of associations (coefficient of correlations), abnormal serum levels of sodium may be one of the most important and useful biochemical marker for predicting poor prognosis.

\section{Acknowledgement:}

We would like to thank the department of neurology at TUTH for helping on these ongoing studies.

\section{References}

1. Adams H P J , Bendixen B H, Kappelle L J. Classification of subtype of acute ischemic stroke. Trial of Org 10172 in Acute Stroke Treatment. Stroke 24 (1): 35-41, 1993

2. Adams H P J, Kappelle L J, Biller J, et al. Ischemic stroke in young adults. Experience in 329 patients enrolled in the Iowa Registry of Stroke in Young Adults. Arch Neurol (52): 491-495, 1995

3. Amarenco P, Bogousslavsky J, Caplan LR, Donnan GA. New approach to stroke subtyping : the ASCO (phenotyping) classification of stroke. Cerebrovascular Dis 27 (5): 502-508, 2009

4. Anastasios Chatzikonstantinou, Marc E. Wolf, Michael G Hennerici. Ischemic stroke in young adults : classification and risk factors. $\mathbf{J}$ neurol 259: 653-659, 2012

5. Bogousslavsky J. Ischemic stroke in patients under age 45. Neurol Clin (10): 113-124, 1992

6. Camerlingo M, Casto L, Censori B, et al. Recurrence after first cerebral infarction in young adults. Acta Neurol Scand (102): 87-93, 2000

7. Carolei A, Marini C,Ferranti E, Frontoni M. A prospective study of cerebral infarction in the young. Analysis of pathogenic determinants. The national research council group. Stroke 24 (3): 362-367, 1993 


\section{Rajbhandari et al}

8. Cerrato P, Grasso M, Imperiale D, Priano 1. Stroke in young patients: etiopathogenesis and risk factors in different age classes. Cerebrovascular Dis 18 (2): 154-159, 2004

9. Correia M, Silva M R, Ferro J M. Prospective community based study of stroke in northern Portugal: incidence and case fatality in rural and urban populations. Stroke 35 (9): 2048-2053, 2004

10. Ferro JM, Massaro A, Mas J. Aetiological diagnosis of ischemic stroke in young adults. Lancet Neurol 9 (11): 1085-1096, 2010

11. K Nedeltchev, $\mathrm{T}$ A der Maur, D Georgiadis, M Arnold, V Caso, H P Mattle, G Schroth, L Remonda, M Sturzenegger, U Fischer, R W Baumgartner . Ischaemic stroke in young adults: predictors of outcome and recurrence. J Neurol Neurosurg Psychiatry (76): 191-195, 2005

12. Kristensen B, Malm J, Carlberg B. Epidemiology and etiology of ischemic stroke in young adults aged 18 to 44 years in Northern Sweden. Stroke 28 (9): 1702-1709, 1997

13. Lee T, Hsu W C, Chen C J. Etiologic study of young ischemic stroke in Taiwan. Stroke 33 (8): 19501955,2002
14. Marini C, Totaro R, Carolei A. Long-term prognosis of cerebral ischemia in young adults. National Research Council Study Group on Stroke in the Young. Stroke (30): 2320-2325, 1999

15. Putaala J, Mesto A, Mesto T M. Analysis of 1008 consecutive patients aged 15 to 49 with first ever ischemic stroke: the Helsinki young stroke registry. Stroke 40 (4): 1195-1203, 2009

16. Rajbhandari R, Gajurel B, Dhungana K, Oli K K. C Reactive Protein in Acute Ischemic Stroke Patients in Tribhuvan University Teaching Hospital. Nepal Journal of Neuroscience 2014, Vol. 11 Issue 1, p4-9. 6p, 2013

17. Truelsen T, Bonita R, Jamrozik K. Surveillance of stroke: A global perspective. Int J Epidemiol (30): S11-6, 2001

18. Varona J F, Guerrra J M, Bernejo F, Molina J A. Causes of ischemic stroke in young adults and evolution of the etiological diagnosis over the long term. Eur Neurol 57 (4): 212-218, 2007

19. Wasay M, Kaul S. Ischemic stroke in young asian women: risk factors and subtypes. Cerebrovascular dis 30 (4): 418-2, 2010 